\title{
Impulsive Noise Cancellation from Cardiac Signal using Modified WLMS Algorithm
}

\author{
Mihir Narayan Mohanty \\ Department of ECE, ITER, SOA University \\ Bhubaneswar, Odisha, India \\ mihir.n.mohanty@gmail.com
}

\author{
Sarthak Panda \\ Department of ECE, ITER, SOA University \\ Bhubaneswar, Odisha, India \\ Sarthak216@rediffmail.com
}

\begin{abstract}
For clean signal, noise cancellation techniques are explored day-by-day. At the user end the clean signal is highly essential for different purposes. In this authors have considered the bio-medical signal that is corrupted with impulsive noise. It is very important to separate from the signal, as its occurrence is sudden and often similar to the signal. The popular adaptive algorithms have been tested for cancellation of impulsive noise. Further most used Wilcoxon LMS is also verified for impulsive noise case. Finally it has been modified for the same purpose. The result found excellent in terms of less MSE, SNR improvement and faster convergence.
\end{abstract}

Index Terms-Impulsive noise; ECG; Adaptive Algorithm; LMS; NLMS; WLMS; MA-WLMS.

\section{INTRODUCTION}

As per World Health Organization review, about 20 million individuals may bite the dust in the year 2017 because of the heart attack. Heart related issues are expanding day by day and Electrocardiogram (ECG) signal is vital in determination of heart related issues. The ECG signal is utilized to know the cardiovascular state of a human. ECG is obtained by the electrical movement of the heart and can be measured by interfacing electrodes on the skin surface of particular parts of the body. As the ECG is a recording of the electrical exercises of the heart, it can help one get an idea of a human's heart exercises and can likewise help in recognizing variations in heart action, for example, cardiovascular infractions or unequal beat intervals.

ECG signal contains various noises. For better decision on patient treatment, it is essential to remove these noised from the monitoring receiver. Due to non-stationary nature of majority ECG signals, filtering of the signal to remove these noised is an essential component of signal processing. Different noises can be associated with ECG signal are: Base line wander, $50 \mathrm{~Hz}$ power line interference, motion artifact electromyogram (EMG) etc. Actually, most sorts of noises are not stationary, it implies, that the noise power measured highlights some variability.

The noise generated by the human muscle is the most difficult noise that ought to be removed. The switching transient in power, incidental pulses in phone lines add to impulsive noises. Such wonders happen in bio-medical signal in diathermia, while utilizing surgical gadgets, in electro cardiology (muscle noise). Also this type of noise gets included in ECG signal at the time of signal acquisition. To accomplish better noise reduction from non-stationary signals like ECG, different adaptive algorithm can be utilized. As adaptive filters don't have fixed channel coefficients, these channels can change their coefficients to lessen the noise present in the signal through adjustment.

A little amount of the works related with this area of investigation has been accomplished. Some major works are cited in the accompanying section. In this piece of work, we have endeavored to develop a system which will nullify the impulsive noise from ECG signal by using adaptive filtering theory. We have modified the Wilcoxon norm based LMS algorithm in a new way to weight variation. We have compared the Signal to Noise ratio (SNR) improvement of various adaptive filters. Also we have compared the Mean Square Error of those filters.

The organization of the paper is as below. The detail literature is provided in section II. In segment III, our proposed method along with some other existing adaptive filtering techniques has been explored. Section IV introduces the results and discussion. Finally, section $\mathrm{V}$ finishes up this paper with conclusion.

\section{RELATED LITERATURE}

Adaptive algorithms have been used in many applications since two to three decades. One of the important applications is noise cancellation. This has been attempted by many researchers also. But the variant of noise with variation of applications are less used till date. Some of the works based on adaptive algorithm for noise cancellation is cited in this section.

Different algorithms concerned to adaptive filter sparsity has been introduced in [1]. The algorithms emphasize on the application domain of echo cancellation and identification of system. It was based on minimization of cost function with respect to a time dependent norm of filter update. They assumed that their problem has not a closed form solution so they have proposed an approximate solution followed by asymptotic behavior. Conventional linear system's performance becomes poor when data is distorted with non- 
Gaussian noise. Adaptive filter design is an alternative solution to it. S.R Kim et. al. have proposed an adaptive preprocessor to minimize the impulsive components when the background noise is correlated with Gaussian process [2]. Their proposed method can adapt to the changes happened in the real time environment and have minimized the effect of impulsive noise from the system. In order to reduce the mixed norms and for the error signals, these authors have proposed two RAPS (robust affine projection sign) algorithms. In RAPS, the norm-based objective function gradient decides the direction vectors. The line search for both RAPS algorithm has been estimated using two norm-based minimization problems. Norm-based direction vector helps minimization of the effect of impulse noise. On the contrary, production of unbiased solution is possible using the line search. The SMAP (set-membership affine projection algorithm is the main source data selective adaptation followed by one of the RAPS technique. As compared to PAPs (pseudo affine projection sign) and Affine projection sign (APS), the RAPS algorithm has shown improvement in steady state misalignment reduction and convergence speed. A simulated application of RAPS has been explored in this area [3]. Similarly the adaptive LMS algorithm was modified to filtered LMS (FxLMS) and used for different applications. But when outliers are present this algorithm does not gives satisfactory result. For improvement of the technique Filtered-X Wilcoxon LMS and Filtered-X least mean log square was proposed. Both of them are basically learning algorithms which are robust in nature [4]. A solution to many problems like target tracking, environment sensing, and data collection is Distributed wireless sensor network. T Panigrahi et. al. have used cost function like Wilcoxon norm and error saturation nonlinearity in impulsive noise environment to solve the robust adaptive estimation problem [5]. But the incremental scheme is not useful to impulsive type environment so that they have used Wilcoxon norm to the incremental scheme to estimate the desired parameters in the existence Gaussian corrupted impulsive noise. LMS algorithm is one of the generally utilized algorithms in many tasks in the field of engineering, medicine, industries etc. The AFA (adaptive filtering algorithm) with averaging is a development over the LMS algorithm and has an enhanced performance. Also authors have used this algorithm in speech processing. Their proposed modification was verified for noise cancellation and had a better performance in terms of improved signal-to-noise ratio [6]. Recovering of signal from distortion and noise is performed by the application of various adaptive algorithms and has made the focus of research work. Excess mean square error is a disadvantage in LMS algorithm that causes performance degradation when desired signal has large power fluctuation. To avoid it, many researchers have given effort in many ways. Some of them have proposed two methods associated to LMS algorithm. One of them is weighted sum method and another is sum method. They have compared the weighted sum method with the sum method. Either one technique provides significant upgrades in the existence of strong desired signals and identical performance in the presence of weak desired signals, with respect to the conventional LMS algorithm [7]. Similarly the averaging algorithm as Filtered-X Adaptive Filtering with Averaging (FxAFA) was used the averages of information and correction term to update the weight value. It has been simulated for single channel feed forward noise controller system and compared their result with FxRLS algorithm on the basis of computational complexity and stability. G.G Yin et. al. have considered the averaging scheme in adaptive algorithm and have obtained optimal convergence with the algorithm with respect to the traditional approach [8-9]. AICF (adaptive impulse correlated filter) for event-related signals was used to remove the noise, even though the noise is colored noise and estimates the deterministic component of the signal. It has experienced with two inputs as primary input and reference input for the performance analysis in terms of signal-to-noise ratio, convergence and misadjustment error applied in ECG signal analysis [10]. RLS algorithm was compared with this algorithm for computational complexity and stability issues. AFA algorithm has high convergence rate as compared to that of the RLS algorithm and low computational complexity and robust in fixed-point calculation. The algorithm was tested in presence of car noise and office noise in speech signal [11]. Widrow's adaptive algorithm have been applied to speech signal, where which there is an acoustic barrier in between primary and reference inputs. During silence active noise canceller can cancel the noise with a little speech distortion by updating the weights. The modified ANC system works well and a SNR improvement up to $11 \mathrm{~dB}$ was achieved by involving a reference input to the outside of the face mask [12]. Comparison among LMS and RLS has been made for $\mathrm{AC}$ and DC noise within ECG signal in [13]. Performance of RLS algorithm is better than that of LMS in terms of MSE and convergence. Also for ECG signal case, M.Z U Rahman et. al. have used normalized signed regressor LMS (NSRLMS) algorithm. This algorithm has less computational complexity due to the sign present in the calculation and better filtering capacity due to the standardized term as compared to the standard algorithms. But it is especially reasonable for applications requiring substantial signal to noise proportions with less computational complexity [14]. Similarly, many trials have been made to cancel the noise from ECG signal with modification of existing algorithms [14-21]. But the literature cannot provide sufficient information regarding impulsive noise cancellation from cardiac signal. For the real time application authors in this piece of work have considered the cardiac signal contaminated with impulsive noise. The proposed algorithm is compared with existing algorithms for its performance and is depicted in the following section.

\section{METHOD FOR IMPULSIVE NOISE CANCELLATION}

The bio-medical signals are recorded with a disturbance at the time of signal acquisition. An extensive variety of noise exists 
in bio-medical signal. One of the noises is a waveform of an electrical movement made by human muscles. In some cases this noise can be impulsive in nature. Impulsive noise can be due to internal i.e. from the human muscle or can be from eternal i.e. from the environment. So separating of this sort of noises is a fundamental need as impulsive noises are sudden burst having high amplitudes. Adaptive filtering theory can be utilized to remove impulsive noise from ECG signal ad adaptive filters can change their coefficients according to the environment.

The proposed block diagram of the adaptive noise cancellation for ECG signal is as follows,

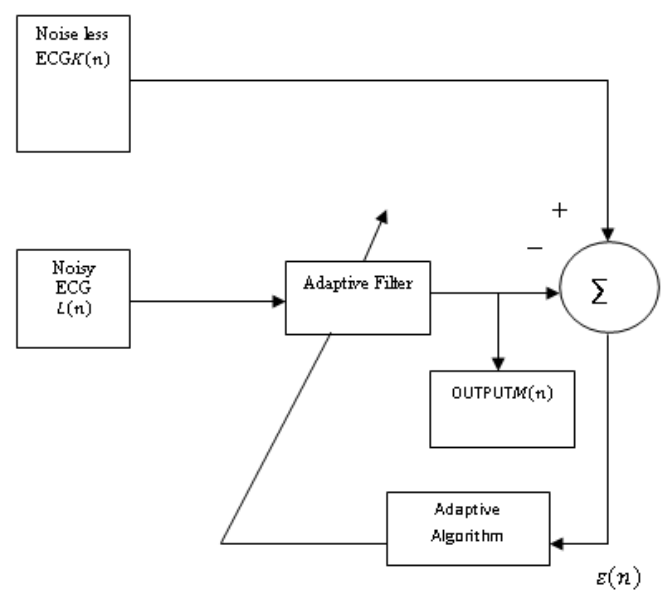

Fig. 1. Structure of Adaptive Noise Cancellation Procedure

Transversal structure is commonly employed framework for adaptive filter implementation. It considered as the desired signal as $K(n)$ which is noise less ECG signal. For input $L(n)$ we have picked an impulsive noise influenced ECG signal. The error signal which is obtained by taking the difference between the desired signal and the output signal gathered by passing the input signal through a adaptive channel is meant by $\varepsilon(n)[22]$.

The error signal $\varepsilon(n)$ can be estimated as,

$$
\varepsilon(n)=K(n)-L(n)
$$

The adaptive changes its weights as demonstrated by the noise signal. Whenever the error will be minimum, ideal point will be reached and at this instance of time we will get our clean ECG signal without impulsive noise. The adaptive output is described by,

$$
M(n)=\omega(n)^{T} L(n)
$$

$\omega(n)$ is the weights of the adaptive filter. Some adaptive algorithms we will explore now to update the weights so as to achieve the noise cancellation task. For comparison purpose, following algorithms have been used. Finally in subsection 3.4 the proposed approach is clearly explained.

\subsection{Least Mean Square (LMS) Algorithm}

When we are analyzing a noisy signal to get the clean signal adaptive algorithm plays a vital role to eliminate the noise. LMS algorithm can be used for changing the weights of the adaptive filter [23-26]. The weight adaption relation can be demonstrated by the following relation [23],

$$
\omega(n+1)=\omega(n)+2 \mu \varepsilon(n) L(n)
$$

Where, $\omega(n+1)$ is the next weight value to be updated and $\mu$ is the convergence coefficient, whose value is generally between 0 to 1 . Also $\mu$ controls the stability of the system and convergence rate.

\subsection{Normalized Least Mean Square (NLMS) Algorithm}

Norm is associated with LMS algorithm. When LMS is normalized, the algorithm is termed as normalized LMS (NLMS). Due to normalization it has a greater stability than LMS algorithm and convergence rate is also higher. The weight update relation of NLMS algorithm can be as follows $[22,23,26]$,

$$
\omega(n+1)=\omega(n)+\mu \cdot \varepsilon(n) \cdot \frac{L(n)}{\delta+\|L(n)\|^{2}}
$$

Here $\delta$ is the small correction factor. Norm can be zero so if the denominator part will be zero then the equation will be invalid. So we are considering one small correction factor to avoid this problem.

\subsection{Wilcoxon Least Mean Square (WLMS) Algorithm}

Let us use the cost function as Wilcoxon norm to upgrade the algorithm using ' $n$ ' number of weights of a linear combiner. The algorithm uses iteration based training. In each iteration ' $\mathrm{m}$ ' samples are used to update the linear combiner weights. A score function is desired to characterize the Wilcoxon norm error term $\varepsilon$ having length ' $x$ '. The conventional LMS can be used to update the model weights so as to expel the norm consistently. The score function can be defined as $[4,5,24,25]$,

$$
\beta(i):[0,1] \rightarrow R
$$

Which is non-diminishing such that,

$$
\int_{0}^{1} \beta^{2}(i) d i<\infty
$$

The score related with the score function $\partial$ can be defined by,

$$
=>d(j)=\beta\left(\frac{j}{l+1}\right)
$$

Where, $\boldsymbol{l}$ is a positive number. It can be demonstrated that the accompanying cost function which is a pseudo norm on $\boldsymbol{R}$.

$$
\begin{aligned}
& J(y)=\|\varepsilon\|_{\omega}=\sum_{k=1}^{l} d\left(\mathrm{R}\left(\varepsilon_{k}\right)\right) \varepsilon_{k} \\
&=\sum_{k=1}^{l} d(k) \varepsilon_{(k)}
\end{aligned}
$$

Where, $\mathrm{R}\left(\boldsymbol{\varepsilon}_{\boldsymbol{k}}\right)$ represents the rank of $\boldsymbol{\varepsilon}_{\boldsymbol{k}}$ among all $\varepsilon_{1}, \ldots, \varepsilon_{l}$. And it can be sort by $\varepsilon_{1}, \leq \cdots \leq, \varepsilon_{l}$ and $d(j)=$ $\boldsymbol{\beta}\left(\frac{j}{l+1}\right)$ and $\boldsymbol{\beta}(\boldsymbol{i})=\sqrt{\mathbf{1 2}}(\boldsymbol{i}-\mathbf{0 . 5})$ Now we can call $\|\boldsymbol{\varepsilon}\|_{\boldsymbol{\omega}}$ as in equation (8) as the Wilcoxon norm of the error vector $\boldsymbol{\varepsilon}$.

To determine the Wilcoxon LMS algorithm we need to use the steepest descent method,

$$
\omega(y+1)=\omega(y)+\mu\left(\nabla_{\omega} J(y)\right)
$$

Where, $\nabla_{\boldsymbol{\omega}} \boldsymbol{J}(\boldsymbol{y})$ can be evaluated as, 


$$
\frac{\partial J(y)}{\partial \omega_{y}}=\sum_{k=1}^{l} d\left(\mathrm{R}\left(\varepsilon_{k}\right)\right) \varepsilon_{k}=\sum_{k=1}^{l} d(k) i_{k}
$$

\subsection{Modified Averaging Wilcoxon Least Mean Square (MA-} WLMS) Algorithm

To reduce the computational complexity and to make the system stable instead of conventional algorithms adaptive algorithm with averaging can be considered. For applications where fast converging is required conventional LMS, NLMS algorithms are not applicable [11]. Also WLMS can be adaptable but it has stability issues. Similarly RLS algorithm can also be adaptable but due to its recursive structure it has more complexity. So in order to overcome all the issues above we have presented a modification to WLMS algorithm based on adaptive filtering with averaging so called Modified Averaging Wilcoxon Least Mean Square (MA-WLMS) algorithm. Adaptive filtering with averaging (AFA) can be realized by as followed [6],

Noise can be estimated as,

$$
N(n)=\sum_{k=0}^{M} \omega(n) N_{1}(n-k)
$$

Where, $M$ is the filter order and $N_{1}$ is the noise component present in the signal.

To minimize the mean square error the filter coefficients are adjusted recursively so as per $[8,11]$ a standard algorithm can be considered for approximating the vector of filter weights as,

$$
\omega(n+1)=\omega(n)+\mu L(n) \varepsilon(n)
$$

Here $L(n)$ is the input signal vector, $\omega(n)$ is the adaptive coefficient vector and $\varepsilon(n)$ is the error vector. $\mu$ is a positive scalar.

Taking the averages of $\omega(n)$ equation (12) can be transformed to,

$$
\omega(n+1)=\overline{\omega(n)}+\frac{1}{n^{\mu}} L(n) \varepsilon(n)
$$

Where we can represent the averages of $\omega(n)$ as follows,

$$
\overline{\omega(n)}=\frac{1}{n} \sum_{k=1}^{n} \omega(n)
$$

The value of $\mu$ lies in between 0.5 to 1 .

According to the analysis present in the upper given algorithm is not stable initially because only the averages of the coefficients have been considered. So to enhance the stability also the averages of the input signal and the error signal is also considered. So the adaptive filtering with averaging can be obtained as,

$$
\omega_{k}(n+1)=\overline{\omega_{k}(n)}+\frac{1}{n^{\mu}} \overline{L(n) \varepsilon(n)}
$$

Where, $k=0,1, \ldots, M$

$\overline{\omega_{k}(n)}$ and $\overline{L(n) \varepsilon(n)}$ can be calculated from their past values, so averaging here does not make extra burden to the calculation. Here covariance matrix is not present so the estimate of covariance is not needed. Automatically computational complexity reduces as well as stability increases.

On application to impulsive noise cancellation from LMS and NLMS algorithm WLMS has better performance. So in order to improve the performance with respect to Signal-toNoise ratio (SNR) we have modified the WLMS algorithm in corporation with adaptive filtering with averaging by introducing the Wilcoxon norm to it. And the Modified Averaging Wilcoxon LMS (MA-LMS) algorithm can be given by,

$$
\omega_{k}(n+1)=\overline{\omega_{k}(n)}+\frac{1}{n^{\mu}} \frac{\overline{L(n) \varepsilon(n)}}{\left\|L_{w}(n)\right\|^{2}}+A V G\left[\frac{1}{n^{\mu}} \frac{\overline{L(n) \varepsilon(n)}}{\left\|L_{w}(n)\right\|^{2}}\right]
$$

The proposed adjustment was evaluated and tested for impulsive noise cancellation in ECG signal.

\section{RESUlT AND DISCUSSION}

For simulation we have acquired the ECG signal from MIT-BIH database [27]. We have tried to cancel out the impulsive noise by considering the following parameters as given in table- -1 .

Table 1 Parameters for Impulsive Noise cancellation

\begin{tabular}{cc}
\hline Parameters & Values \\
\hline $\boldsymbol{\mu}$ for adaptive algorithms & 0.08 \\
$\boldsymbol{\delta}$ for NLMS algorithm & 0.001 \\
No. of iteration & 3600 \\
Tap-weight & 16 \\
\hline
\end{tabular}
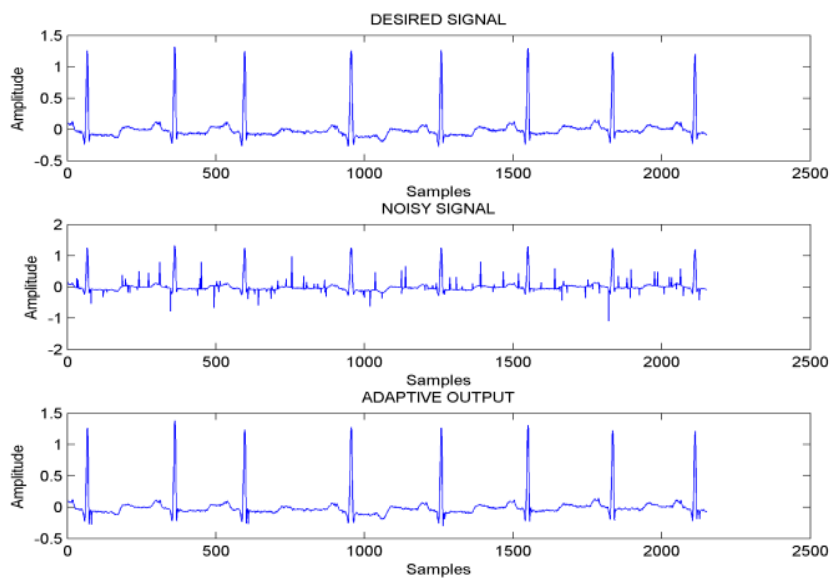

Fig. 2. Impulsive noise cancellation using LMS algorithm
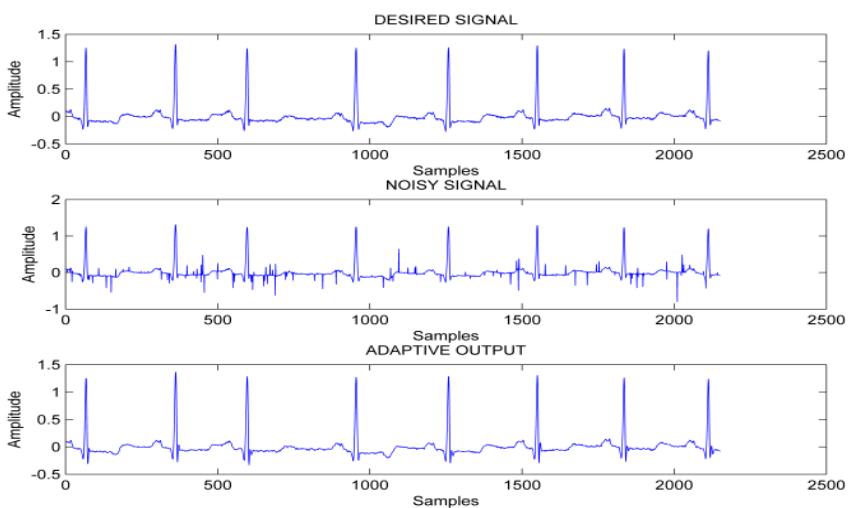

Fig. 3. Impulsive noise cancellation using NLMS algorithm 

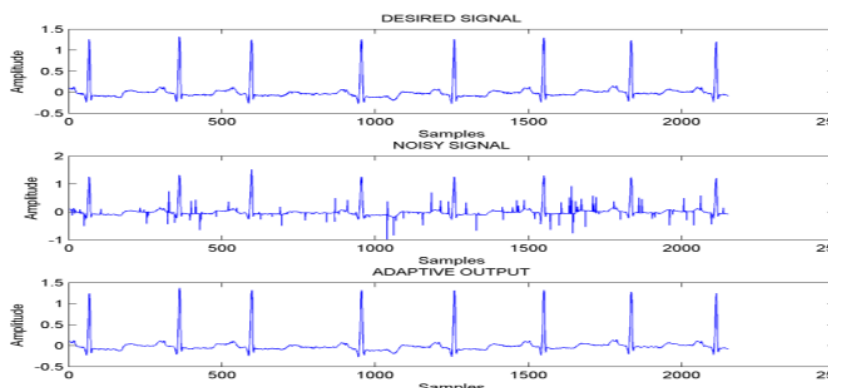

Fig. 4. Impulsive noise cancellation using WLMS algorithm
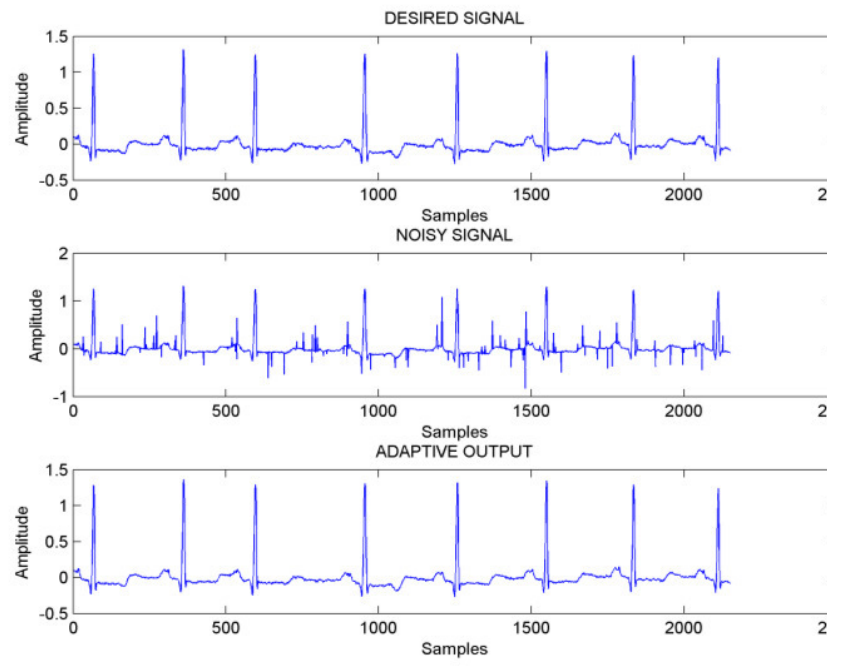

Fig. 5. Impulsive noise cancellation using MA-WLMS algorithm

Fig.2 to Fig.5 demonstrates the impulsive noise cancellation from ECG sign utilizing LMS, NLMS,WLMS and MA-WLMS algorithm separately. In all cases noise is removed so to know which calculation is better we have compared the Mean Square curve of each algorithm.

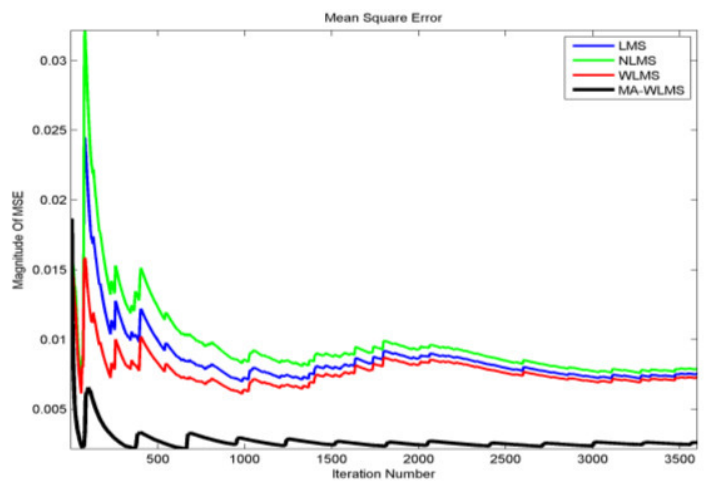

Fig.6. MSE comparison between LMS, NLMS, WLMS and MA-WLMS algorithm.

Also we have obtained the performance of the proposed algorithm with respect to SNR.SNR before filtering and SNR after filtering has beeen calculated an. At that point we have also calulated the SNR improvement.From Table.2 MA-
WLMS algorithm has preferred SNR improvement than LMS, NLMS and WLMS.

Table 2: SNR comparison between LMS, NLMS, WLMS and MAWLMS

\begin{tabular}{cccc}
\hline $\begin{array}{c}\text { FILTER } \\
\text { TYPE }\end{array}$ & $\begin{array}{c}\text { SNR } \\
\text { BEFORE } \\
\text { FILTERING }\end{array}$ & $\begin{array}{c}\text { SNR AFTER } \\
\text { FILTERING }\end{array}$ & $\begin{array}{c}\text { SNR } \\
\text { IMPROVEMENT }\end{array}$ \\
\hline LMS & $7.8499 \mathrm{~dB}$ & $11.3658 \mathrm{~dB}$ & $3.5159 \mathrm{~dB}$ \\
NLMS & $7.0176 \mathrm{~dB}$ & $12.5551 \mathrm{~dB}$ & $5.5375 \mathrm{~dB}$ \\
WLMS & $7.1397 \mathrm{~dB}$ & $15.4473 \mathrm{~dB}$ & $8.3076 \mathrm{~dB}$ \\
MA- & $7.1086 \mathrm{~dB}$ & $16.8498 \mathrm{~dB}$ & $9.7412 \mathrm{~dB}$ \\
WLMS & & & \\
\hline
\end{tabular}

\section{CONCLUSION}

The proposed algorithm has shown excellency for noise removal. Though impulsive noise is complex, it is removed using the proposed algorithm and the SNR has been improved almost three times than standard LMS algorithms. It is suitable for the sensitive bio-medical applications. The algorithm may be verified for other signals and can be suitably used for real time application. The implementation of the algorithm can be useful for modern medical equipments and can be extended for future work.

\section{References}

[1] Vega L, Rey H, Benesty J, Tressens S. A Family of Robust Algorithms Exploiting Sparsity in Adaptive Filters. IEEE Transactions on Audio, Speech, and Language Processing. 2009; 17(4): 572-81.

[2] Seong Rag KimEfron A. Adaptive robust impulse noise filtering. IEEE Transactions on Signal Processing. 1995; 43(8): 1855-66.

[3] Bhotto M, Ahmad M, Swamy M. Robust Shrinkage Affine-Projection Sign Adaptive-Filtering Algorithms for Impulsive Noise Environments. IEEE Transactions on Signal Processing. 2014; 62(13): 3349-59.

[4] Vasundhara, Panda G, Puhan NB. Robust feedback cancellation in hearing aids in the presence of outliers. International Conference on Signal Processing and Integrated Networks (SPIN) 2014; 25-29.

[5] Panigrahi T, Panda G, Mulgrew B, Majhi B. Robust Incremental LMS over Wireless Sensor Network in Impulsive Noise. International Conference on Computational Intelligence and Communication Networks 2010; 205-09.

[6] Vijaykumar VR, Vanathi PT, Kanagasapabathy P. Modified Adaptive Filtering Algorithm for Noise Cancellation in Speech Signals. Electronics and Electrical Engineering, Elektronika IR Elektrotechnika 2007; 2(74): 17-20.

[7] Greenberg J. Modified LMS algorithms for speech processing with an adaptive noise canceller. IEEE Transactions on Speech and Audio Processing. 1998; 6(4): 338-51.

[8] Akhtar M, Abe M, Kawamata M. Adaptive Filtering With AveragingBased Algorithm for Feed forward Active Noise Control Systems. IEEE Signal Processing Letters. 2004; 11(6): 557-60.

[9] Yin G, Zhu Y. Averaging procedures in adaptive filtering: an efficient approach. IEEE Transactions on Automatic Control. 1992; 37(4): 46675.

[10] Laguna P, Jane R, Meste O, Poon P, Caminal P, Rix H et al. Adaptive filter for event-related bioelectric signals using an impulse correlated reference input: comparison with signal averaging techniques. IEEE Transactions on Biomedical Engineering. 1992; 39(10): 1032-44.

[11] Iliev G, Kasabov N. Adaptive filtering with averaging in noise cancellation for voice and speech recognition. Department of Information Science, University of Otago; 2001. 
[12] Harrison W, Jae Lim, Singer E. A new application of adaptive noise cancellation. IEEE Transactions on Acoustics, Speech, and Signal Processing. 1986; 34(1): 21-27.

[13] Islam S, Islam SZ, Jidin R, Ali M. Performance Study of Adaptive Filtering Algorithms for Noise Cancellation of ECG Signal. $7^{\text {th }}$ International Conference on Information, Communications and Signal Processing 2009: 1-5.

[14] Rahman MZU, Shaik RA, Reddy DVR. An Efficient Noise Cancellation Technique to remove noise from the ECG signal using Normalized Signed Regressor LMS algorithm. International Conference on Bioinformatics and Biomedicine 2009: 257-60.

[15] Thakor N, Zhu Y. Applications of adaptive filtering to ECG analysis: noise cancellation and arrhythmia detection. IEEE Transactions on Biomedical Engineering. 1991; 38(8): 785-94.

[16] Yelderman M, Widrow B, Cioffi J, Hesler E, Leddy J. ECG Enhancement by Adaptive Cancellation of Electrosurgical Interference. IEEE Transactions on Biomedical Engineering. 1983; BME-30(7): 39298.

[17] Rahman M, Shaik R, Reddy D. Efficient and Simplified Adaptive Noise Cancelers for ECG Sensor Based Remote Health Monitoring. IEEE Sensors J. 2012; 12(3): 566-73.

[18] Lu G, Brittain J, Holland P, Yianni J, Green A, Stein J et al. Removing ECG noise from surface EMG signals using adaptive filtering. Neuroscience Letters. 2009; 462(1):14-19.

[19] Joshi V, Verma A, Singh Y. De-noising of ECG Signal Using Adaptive Filter Based on MPSO. Procedia Computer Science. 2015; 57: 395-02.
[20] Mirza A, Kabir S, Ayub S, sheikh S. Impulsive Noise Cancellation of ECG signal based on SSRLS. Procedia Computer Science. 2015; 62: 196-02.

[21] AlMahamdy M, Riley H. Performance Study of Different Denoising Methods for ECG Signals. Procedia Computer Science. 2014; 37: 325 32.

[22] Widrow B, Stearns S. Adaptive signal processing. Englewood Cliffs, N.J.: Prentice-Hall; 1985.

[23] Haykin S. Adaptive filter theory. Englewood Cliffs, N.J.: Prentice-Hall; 1986.

[24] Dash S, Mohanty MN. Analysis of outliers in system identification using WLMS algorithm. International Conference on Computing, Electronics and Electrical Technologies (ICCEET) 2012;: 802-06.

[25] Panda S, Mohanty MN. Analysis of Norms in Adaptive algorithm On Application of System Identification. Information Systems Design and Intelligent Applications, Advances in Intelligent Systems and Computing. 2016; 433: 579-88.

[26] Panda S, Mohanty MN. Impulsive Noise Cancellation from ECG signal using Adaptive Filters and their Comparison. Indonesian Journal of Electrical Engineering and Computer Science.2016; 3(2):281-88.

[27] PhysioBank ATM [Internet]. Physionet.org. 2016 [cited 8 August 2016]. Available from: https://www.physionet.org/cgi-bin/atm/ATM. 